\title{
Design and Simulation of a Novel Hybrid Leg Mechanism for Walking Machines
}

\author{
M. Demirel ${ }^{1}$, G. Carbone ${ }^{2}$, M. Ceccarelli ${ }^{3}$ and G. Kiper ${ }^{4}$ \\ 1 "Murat Demirel" RAML: Rasim Alizade Mechatronics Laboratory, Izmir \\ Institute of Technology,Turkey,e-mail: muratdemirel@iyte.edu.tr \\ 2 "'Giuseppe Carbone" LARM: Laboratory of Robotics and Mechatronics, \\ DiMSAT - University of Cassino, Italy, e-mail: carbone@unicas.it \\ 3 "Marco Ceccarelli" LARM: Laboratory of Robotics and Mechatronics, DiMSAT \\ - University of Cassino, Italy, e-mail: ceccarelli@unicas.it \\ 4 "Gökhan Kiper" RAML: Rasim Alizade Mechatronics Laboratory, Izmir \\ Institute of Technology,Turkey,e-mail: gokhankiper@iyte.edu.tr
}

\begin{abstract}
.
This paper introduces a novel hybrid structure design that is composed of rigid links and cables for a robotic leg with static walking. The proposed mechanism is characterized by actuated hip joints, passive knee joints and an actuated prismatic foot joint. The foot is the moving platform of the proposed mechanism which possesses pure translational motion due to the passive parallelograms with cables. Kinematic analysis has been worked out for evaluating a typical human-like gait trajectory. A 3-D model has been developed and simulation are made in SolidWorks ${ }^{\circledR}$ environment. Simulation results show that the proposed mechanism is able to perform an ovoid walking cycle of a foot point and the computed actuator torques and forces are in a feasible range for a low-cost and easy-operation design. The simulation results will be used for a prototype construction in a future work.
\end{abstract}

Key words: Walking machines, Hybrid mechanisms, Leg mechanisms, Static walking simulation.

\section{Introduction}

Walking machines are of special interest to researchers since the beginning of the technology of transportation machinery due to their better obstacle avoidance capability than wheeled systems [1, 2]. Most of the existing biped robots are based on serial kinematic architectures with 6-degrees of freedom (dof) legs, such as [3, $4,5,8,11]$. There are some other walking machines with linkage mechanisms [1, 2]. If human anatomy is considered, human leg movement can be described as parallel manipulator because of the muscular system of a leg [6]. Parallel manipu- 
lators have good performance in terms of accuracy, rigidity and payload to weight ratio [7]. However, their workspaces are more limited than serial manipulators. Walking machines with parallel manipulators have successful examples, such as $[9,10]$.

New leg architectures with less than 6-dof are suggested in [6] which are composed of rigid links and cables (hybrid structures). It is shown that a human leg 6dof is required for rough terrain adaptability. Less than 6-dof parallel manipulators can be used as leg design for flat surface applications, since orientation dofs are not required for these applications. Even turning requirement of leg can be solved by waist rotation [1]. Walking machines with reduced numbers of dof leg architectures still need to be investigated with new solutions.

In this paper, a novel hybrid structure leg design with 3 translational dofs is proposed. Kinematic analysis of the proposed architecture has been worked out for evaluating a typical human-like gait trajectory. Kinematic simulation of a threedimensional (3-D) model has been computed in Solidworks ${ }^{\circledR}$ environment with the aim to evaluate the operation performances of proposed hybrid leg mechanism for walking machines.

\section{Configuration and a CAD Model}

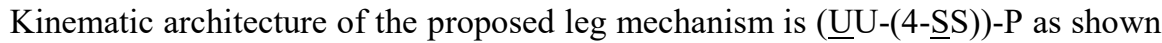
in Fig. 1 where underlined joints $\underline{U}$ and $\underline{S}$ represent fixed joints. This mechanism consists of a fixed base (hip), a middle platform (knee), a moving platform

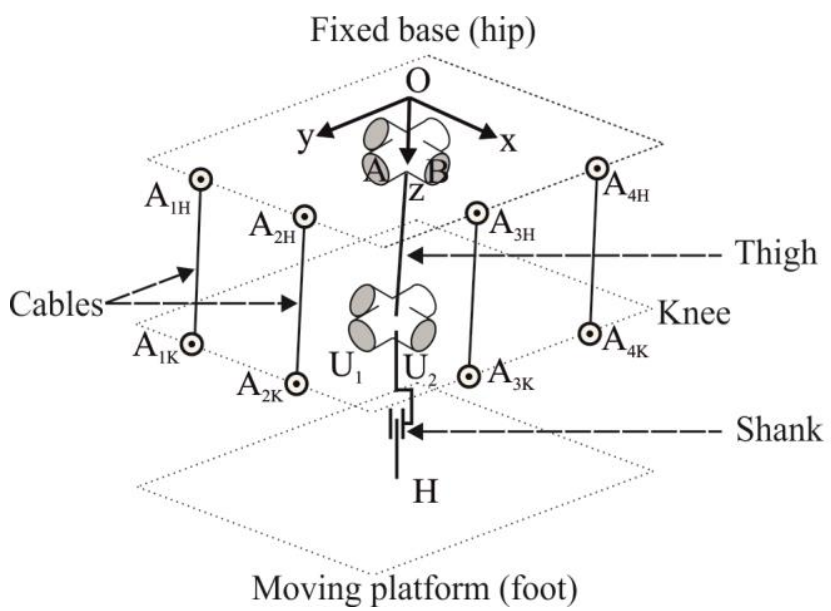

(a)

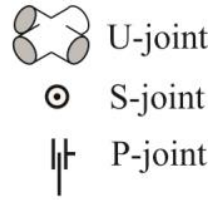

(b)

Fig. 1 Kinematic architecture of the proposed mechanism; (a) a scheme, (b) schemes of the used constraints 
(foot), two rigid links (thigh and shank) and four passive cables. The thigh connects the hip to the knee by two universal joints (U-Joint) at each end. The shank connects the knee to the foot by a prismatic joint (P-joint). Each passive cable is equivalent to a rigid link and it has spherical joints (S-joint) at each end. UU link provides a rotational and a translational constraint to the knee. Two cables make a parallelogram loop which also provide one rotational constraint to the knee. Hence, combination of UU link and two parallelogram loops ensures to constrained the orientation of the foot. That is, the foot has pure translational motion. $\mathrm{U}$ joint on the hip (rotary actuators A and B, see Fig. 2-(a)) and prismatic joint (linear actuator) between the knee and the foot are actuated.

A 3-D model of the proposed leg mechanism is designed in SolidWorks ${ }^{\circledR}$ environment as shown in Fig. 2-(a). The main specifications of the proposed mechanism are listed in Table 1. Dimension parameters are indicated and listed in Fig. 2(b), Fig. 2-(c) and Table 2, respectively. The distance between the actuated U-joint centers and anchor points of cables $\left(\mathrm{A}_{1 \mathrm{H}}, \mathrm{A}_{2 \mathrm{~K}}\right.$, etc.) are called as $\mathrm{d}_{\mathrm{UH}}, \mathrm{d}_{\mathrm{UK}}$ and they should be equal to each other so that the cable lengths are kept constant and the platform possesses pure translational motion. Eight cables are used in the CAD model instead of four due to the kinematic constraint requirements of the CAD program. Mass values of cables are almost negligible, and therefore addition of four cables does not contribute as additional mass in the simulation. Since cables are passive, they can be installed to the platforms without pulleys. Cables are

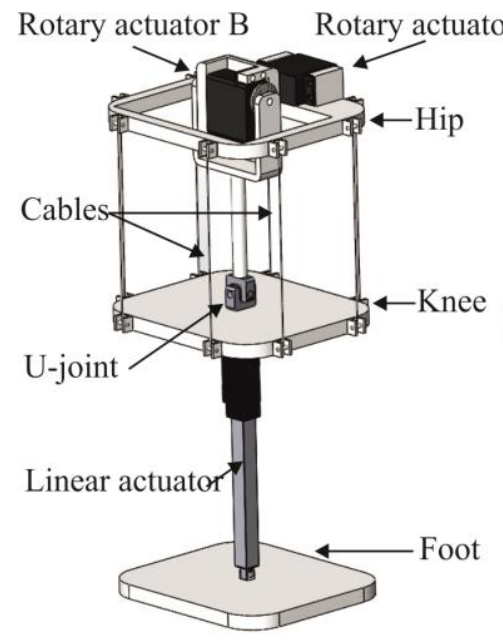

(a)

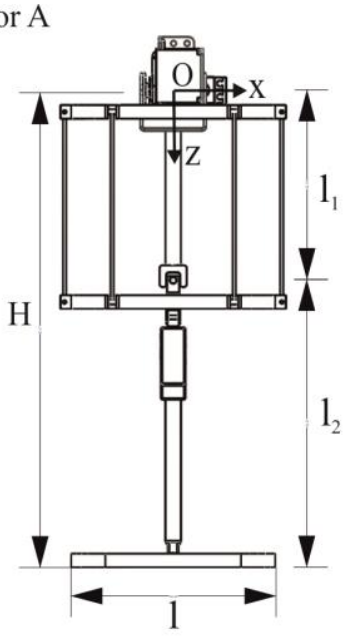

(b)

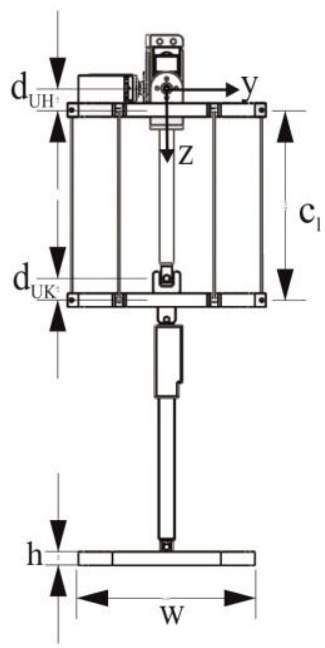

(c)

Fig. 2 Proposed leg mechanism; (a) CAD model, (b) dimension parameters of the CAD model from sagittal view (xz plane) (c) front view (yz plane) 
modeled as cylindrical links by assuming they are always in tension. Angular displacements of the rotary actuators $A$ and $B$ are denoted as $\theta_{1}$ and $\theta_{2}$, where displacement of linear actuator is $\mathrm{L}_{2}$. $\mathrm{L}_{2}$ can be expressed in Eq. (1) as the sum of initial length $\left(l_{2}\right)$ and stroke $(s)$,

$$
L_{2}=l_{2}+s
$$

Table 1. Main specifications of the proposed mechanism in Fig. 2

\begin{tabular}{ccccc}
\hline DOF & Weight $(\mathrm{kg})$ & Dimension $(\mathrm{mm})$ & Step size $(\mathrm{mm})$ & Step cycle $(\mathrm{s})$ \\
\hline 3 & 0.75 & $150 \times 130 \times 351$ & $200 \times 50$ & 1 \\
\hline
\end{tabular}

Table 2. Dimension parameters (in $\mathrm{mm}$ ) and mass values (in grams) of the model in Fig. 2

\begin{tabular}{cccccccc}
\hline$l$ & $w$ & $h$ & $l_{l}$ & $l_{2}$ & $d_{u}$ & $c_{l}$ & $H$ \\
\hline 150 & 130 & 10 & 140 & 211 & 11 & 140 & 351 \\
\hline
\end{tabular}

Table 3. Mass values (in grams) of the CAD model

\begin{tabular}{cccccc}
\hline$m_{H}$ & $m_{K}$ & $m_{F}$ & $m_{U}$ & $m_{L 1}$ & $m_{A}$ \\
\hline 95 & 195 & 195 & 12 & 74 & 80 \\
\hline
\end{tabular}

Mass centers of each platform are set in geometric center of the platforms. Mass values of each component are listed in Table 3, as hip platform $\left(\mathrm{m}_{\mathrm{H}}\right)$, knee platform $\left(\mathrm{m}_{\mathrm{K}}\right)$, foot $\left(\mathrm{m}_{\mathrm{F}}\right)$, universal joint $\left(\mathrm{m}_{\mathrm{U}}\right)$, thigh $\left(\mathrm{m}_{\mathrm{L} 1}\right)$, rotary actuators $\left(\mathrm{m}_{\mathrm{A}}\right)$, linear actuator $\left(\mathrm{m}_{\mathrm{L}}\right)$. The torques of rotary actuator are denoted as $\tau_{1}$ and $\tau_{2}$, where the force of linear actuator is $F_{L}$. Feasibility of the proposed design is ensured by selecting light-weight materials for the platforms (like ABS) and commercial products (U-joint, linear actuator and rotary actuators). These components are modelled properly in a 3-D model for static walking simulation.

\section{Simulation Model and Results}

The aim of this paper is to confirm feasibility of the proposed hybrid leg architecture for walking machine. Therefore, a kinematic analysis of a human-like walking trajectory is carried out through for the simulation. This trajectory is an ovoid curve as shown in Fig. 3-(a) and it is composed of two phases called as swinging and supporting phases. The straight-line segment represents the supporting phase where the curved segment represents the swinging phase. Two length parameters are enough to describe step cycle as step length $\left(\mathrm{S}_{\mathrm{L}}\right)$ and step height $\left(\mathrm{S}_{\mathrm{H}}\right)$. Walking gait of foot point $\mathrm{H}$ is defined as A-B-C-D-A for $1 \mathrm{~s}$ step cycle. Displacement of foot point is shown in Fig. 3-(b) a for walking cycle $S_{L}=200 \mathrm{~mm}$ and $S_{H}=50$ 


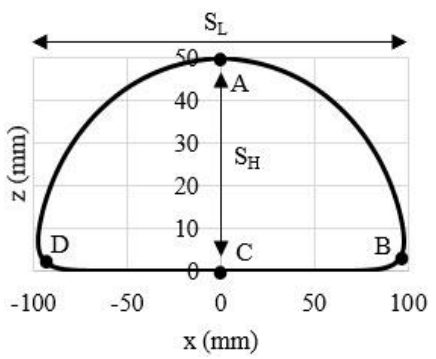

(a)

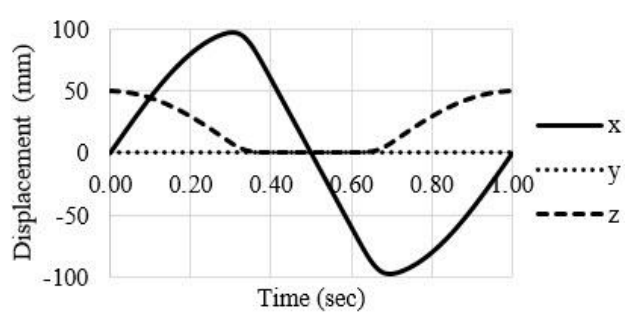

(b)

Fig. 3 Walking cycle; (a) ovoid curve cycle for $\mathrm{SH}=50, \mathrm{SL}=200 \mathrm{~mm}$, (b) displacement of foot point $\mathrm{H}$

$\mathrm{mm}$. Static walking simulation requires only motions of the rotary actuator $\mathrm{A}\left(\theta_{1}\right)$ and the linear actuator $(\mathrm{s})$. Rotary actuator $\mathrm{B}$ has no motion $\left(\theta_{2}=0\right)$ in the planned trajectory. $\theta_{1}$ and $\mathrm{s}$ values are calculated by using inverse kinematics equations through Eqs. (2)-(3) according to the position of the foot point $\mathrm{H}$ in reference frame $(\mathrm{O}, \mathrm{x}, \mathrm{y}, \mathrm{z})$.

$$
\begin{gathered}
\theta_{1}=\arcsin \left(\frac{x_{P}}{l_{1}}\right) \\
s=z_{P}-l_{1} \cdot \cos \left(\theta_{1}\right)-l_{2}
\end{gathered}
$$

For a walking cycle of three steps in 3 seconds, input position data of rotary actuator $\mathrm{A}$ and linear actuator are shown in Fig. 4. In particular, it is found, $\Delta \theta_{1}=$ $\pm 42^{\circ}$ and $\Delta \mathrm{s}=0-72 \mathrm{~mm}$. Simulation of three steps is performed in SolidWorks ${ }^{\circledR}$ environment by using "Motion Analysis" toolbox. This toolbox uses MSC Adams ${ }^{\circledR}$ solver. Snapshots of the first step cycle for points $A(t=0 s)-B(t=0.33 s)$ $-\mathrm{C}(\mathrm{t}=0.5 \mathrm{~s})-\mathrm{D}(\mathrm{t}=0.66 \mathrm{~s})-\mathrm{A}(\mathrm{t}=1 \mathrm{~s})$ are shown in Fig. 5 from sagittal view.

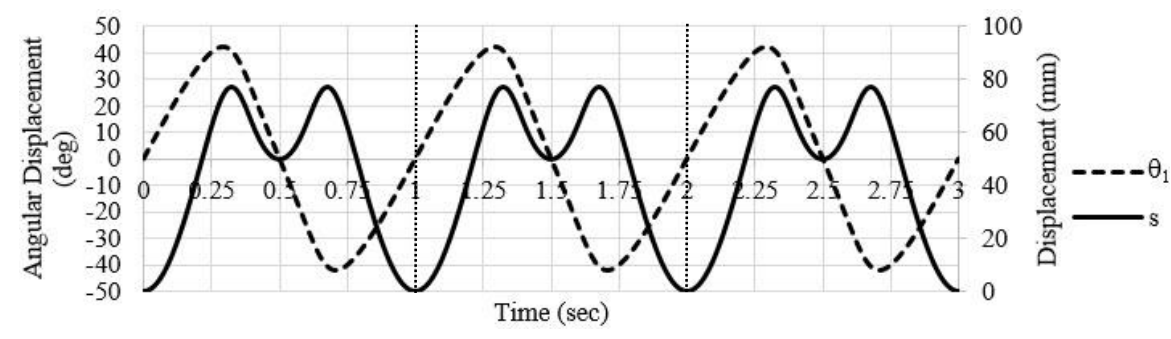

Fig. 4 Input angular displacement of rotary actuator $\mathrm{A}\left(\theta_{1}\right.$ vs time) and input displacement of the linear actuator (s vs time) 


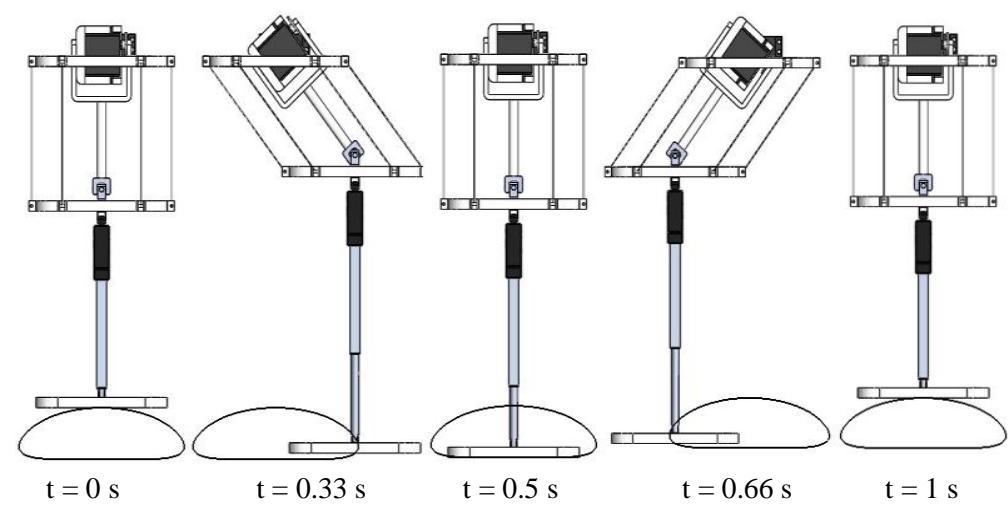

Fig. 5 Snapshots of the simulated motion in xz-plane

Computed $\tau_{1}$ and $F_{L}$ values of the simulation are shown in Fig. 6 . Maximum torque and force values of actuators are required at the step phase changes, point $\mathrm{B}$ and $\mathrm{D}: \tau_{1 \max }=0.271 \mathrm{~N} \cdot \mathrm{mm}$ and $\mathrm{F}_{\mathrm{Lmax}}=2.6 \mathrm{~N}$. Computed joint reaction forces of hip joints $A$, $B$ and knee joint $U_{1}$ in $\mathrm{X}-$, $y$ - and z-directions are shown in Fig. 7. Some numerical errors can be seen in Fig. 7-a for $F_{A Z}$ and $F_{B Z}$. Maximum joint reaction forces are about $6 \mathrm{~N}$ in all revolute joints. Since total weight of the mechanism is about $7.5 \mathrm{~N}$, the computed reaction forces are reasonable. Note that reaction forces are computed without friction.

As mentioned before, mass centers of the platforms are chosen as the geometric centers of the platforms. Acceleration values of these points in $\mathrm{x}$ - and $\mathrm{z}$-directions are shown in Fig. 8. Maximum speed and acceleration values of center of masses of the knee and the foot are computed as $\mathrm{V}_{\mathrm{KX}}=$ $611 \mathrm{~mm} / \mathrm{s}, V_{\mathrm{KZ}}=305 \mathrm{~mm} / \mathrm{s}, \mathrm{a}_{\mathrm{KX}}=6669 \mathrm{~mm} / \mathrm{s}^{2}, \mathrm{a}_{\mathrm{KZ}}=0.2 \mathrm{~mm} / \mathrm{s}^{2}$ and $\mathrm{V}_{\mathrm{FX}}$ $=611 \mathrm{~mm} / \mathrm{s}, V_{\mathrm{FZ}}=204 \mathrm{~mm} / \mathrm{s}, \mathrm{a}_{\mathrm{FX}}=6669 \mathrm{~mm} / \mathrm{s}^{2}, \mathrm{a}_{\mathrm{FZ}}=2044 \mathrm{~mm} / \mathrm{s}^{2}$. Since there is only translational motion in $\mathrm{z}$-direction between the knee and the foot platforms, $\mathrm{a}_{\mathrm{KX}}$ and $\mathrm{a}_{\mathrm{FX}}$ values are equal during the simulation. Maximum values are occurring in step phase changes (point B and D) again. These sudden changes are due to inertial effects of the mechanism.

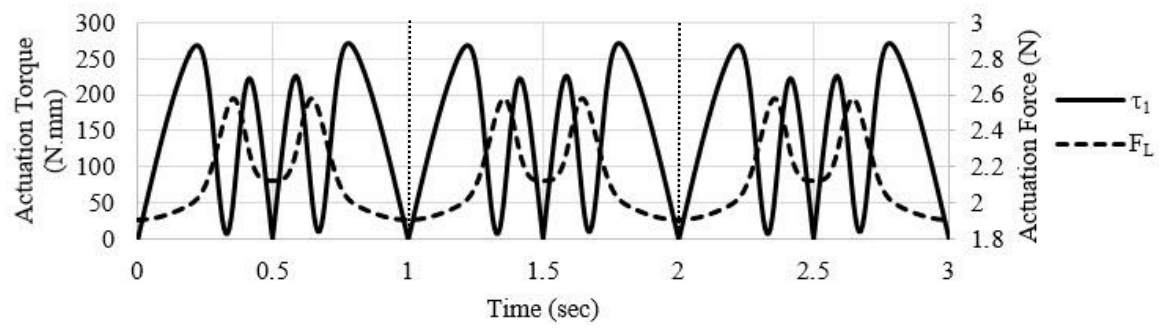

Fig. 6 Computed actuation torque of rotary actuator $A\left(\tau_{1}\right)$ and force of linear actuator $\left(\mathrm{F}_{1}\right)$ 


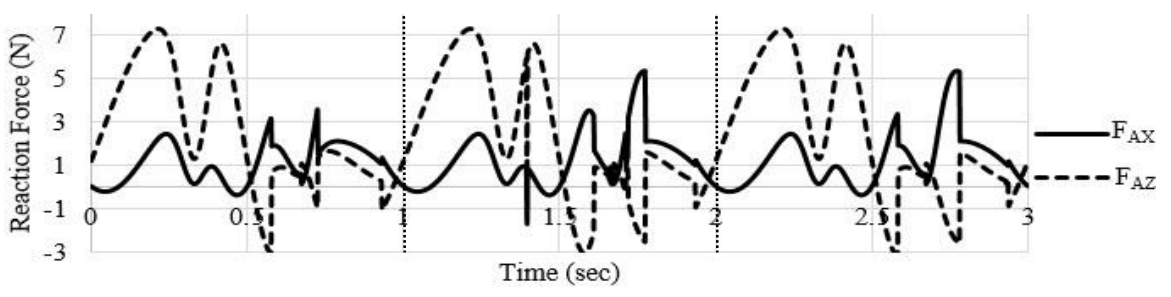

(a)

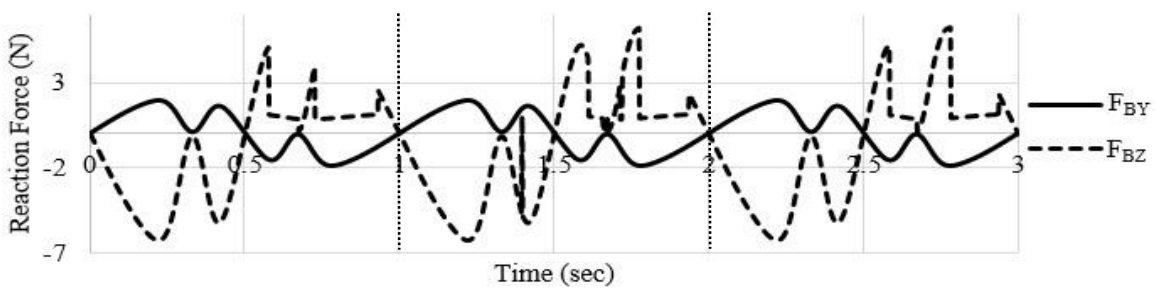

(b)

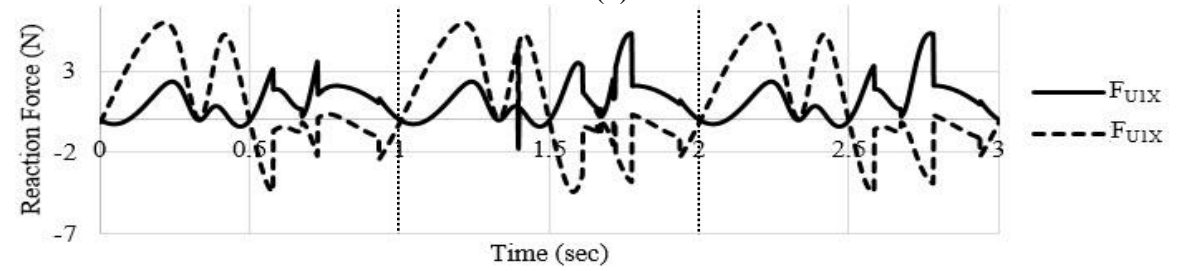

(c)

Fig. 7 Computed reaction forces of the joints; (a) hip joint A FAX, $F_{A Z}$ (b) hip joint $B$ FBY, $F_{B Z}(c)$ knee joint $U_{1}$ FU1X, FU1Z

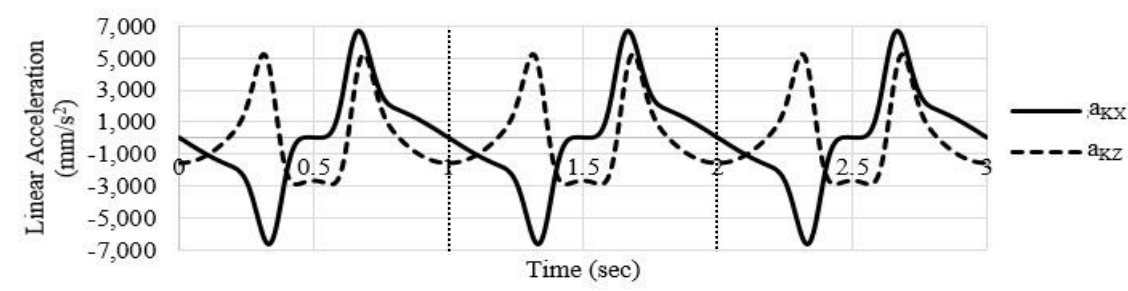

(a)

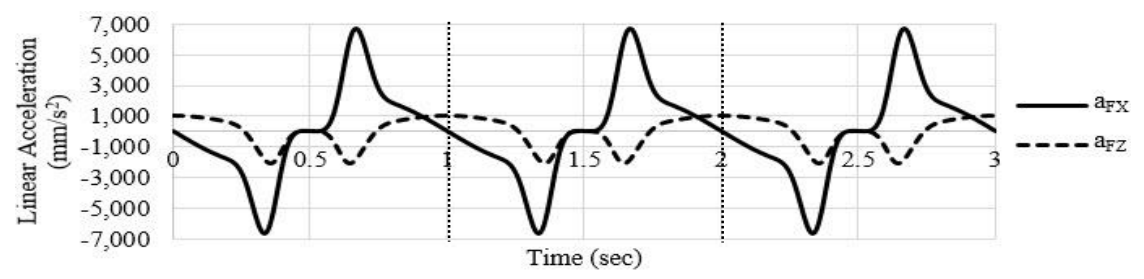

(b)

Fig. 8 Computed center of mass accelerations in Ox and Oz direction; (a) the knee point, (b) the foot point 


\section{Conclusions}

In this paper, a novel hybrid leg mechanism is presented for walking machines. Kinematic analysis of the proposed leg mechanism has been worked out to characterize the operation performance. Static walking simulation is computed in Solidworks ${ }^{\circledR}$ environment and simulation results show that the proposed leg mechanism is able to accomplish a human-like foot step trajectory. Computed torque and force values of the actuators and reaction forces ensure feasibility of the proposed design for a low-cost design. The simulation results will be used for a prototype construction in future work.

Acknowledgments Part of this work was developed during an Erasmus visit of the first author at LARM: Laboratory of Robotics and Mechatronics, Cassino University, Italy in 2017.

\section{References}

1. Carbone G. and Ceccarelli M., Legged robotic systems, Cutting Edge Robotics, Wien-New York, pp. 553-577 (2005)

2. Ceccarelli, M. and Kececi, F.. Designs and Prototypes of Mobile Robots. Momentum Press. (2015)

3. Kaneko K., Kanehirao F., Morisawa M., Miura K., Nakaoka S. I. and Kajita S., Cybernetic human HRP-4C, Humanoid Robots, 9th IEEE-RAS International Conference, pp. 7-14 (2009)

4. Kondo H., Morishima A., Ogura Y., Momoki S., Shimizu J., Lim H. O. and Takanishi A., Algorithm of pattern generation for mimicking disabled person's gait, Biomedical Robotics and Biomechatronics, 2nd IEEE RAS and EMBS International Conference, pp. $724-729$ (2008)

5. Lohmeier S., Buschmann T. and Ulbrich H., Humanoid robot LOLA, Robotics and Automation ICRA'09, IEEE International Conference, pp.775-780 (2009)

6. Marco C., and Giuseppe C., A new leg design with parallel mechanism architecture, In Advanced Intelligent Mechatronics, IEEE/ASME International Conference, pp. 1447-1452 (2009)

7. Merlet, J.-P.: Parallel Robots (Series: Solid Mechanics and Its Applications). Springer (2006)

8. Rocchi A., Hoffman E. M., Fanioli E. and Tsagarakis N. G., A whole-body stack-of-tasks compliant control for the humanoid robot COMAN (2015)

9. Sugahara Y., Endo T., Lim H. O. and Takanishi A., Design of a battery-powered multipurpose bipedal locomotor with parallel mechanism, Intelligent Robots and Systems, Vol. 3, IEEE/RSJ International Conference, pp. 2658-2663 (2002)

10. Wang, M., Ceccarelli, M., and Carbone, G., A feasibility study on the design and walking operation of a biped locomotor via dynamic simulation. Frontiers of Mechanical Engineering, 11(2), pp. 144-158 (2016).

11. Yu Z., Huang Q., Ma G., Chen X., Zhang W., Li J. and Gao J., Design and development of the humanoid robot BHR-5, Adcances in Mechanical Enginnering, Vol. 6 (2014) 